\title{
Artikel 94 B-VG und die Errichtung des österreichischen Abrechnungsgerichtshofs
}

It used to be commonly held that until a recent amendment of article 94, the Austrian constitution prevented the courts of justice from deciding on appeals against administrative decisions. This view has however been challenged with the suggestion that this particular interpretation of article 94 did not develop until more than thirty years after the constitution was enacted in 1920. If this theory is correct, one would expect to find some historical evidence of courts of justice that specifically served as appellate instances in administrative matters. Such a court was the 'Abrechnungsgerichtshof' (Clearing Court) that existed in Vienna from 1922 to 1944. The present contribution investigates how contemporary scholarship and the judicature of the Constitutional Court characterised this court in regard to article 94.

\section{Einleitung: Art. 94 B-VG und das Problem der Instanzenzüge}

Die Verwaltungsgerichtsbarkeits-Novelle 2012, BGB1 I 51/2012, die am 1. Jänner dieses Jahres in Kraft getreten ist, hat vor allem wegen der Abschaffung des administrativen Instanzenzuges und der damit einhergehenden Einführung von neun Landes- und zwei Bundesverwaltungsgerichten Beachtung gefunden. Weniger öffentlichkeitswirksam war hingegen die Änderung des Art. 94 B-VG durch dieselbe Novelle. Dieser Artikel hatte bis dahin knapp und präzise gelautet: „Die Justiz ist von der Verwaltung in allen Instanzen getrennt." Nunmehr sieht ein neu eingefügter Abs. 2 vor, dass durch einfaches Bundes- oder Landesgesetz ,in einzelnen Angelegenheiten anstelle der Erhebung einer Beschwerde beim Verwaltungsgericht ein Instanzenzug von der Verwaltungsbehörde an die ordentlichen Gerichte vorgesehen werden" kann. Nach den Materialien soll es dem einfachen Gesetzgeber damit ermöglicht werden,
Ausnahmen vom Grundsatz der Trennung von Justiz und Verwaltung zu schaffen. ${ }^{1}$ Gäbe es den neuen Art. 94 Abs. 2 B-VG nämlich nicht, wäre ein Instanzenzug von einer Verwaltungsbehörde an ein ordentliches Gericht nach der herrschenden Lehre und Rechtsprechung wegen Verletzung des Trennungsgrundsatzes verfassungswidrig und damit unzulässig. Begründet wurde diese Auffassung mit den Argumenten, dass der Instanzenzug aufgrund der verfahrensrechtlichen Verflechtung der getrennt zu haltenden Gewalten eine verpönte organisatorische Einheit herstelle, ${ }^{2}$ bzw. dass der Gesetzgeber verhalten sei, jede Materie in ihrer Gesamtheit entweder den Verwaltungsbehörden oder den Gerichten zur Vollziehung zuzuweisen. ${ }^{3}$

Der vorliegende Aufsatz geht der Frage nach, ob diese bis zur Novelle dominante Auffassung des Trennungsgrundsatzes, die einen Instanzenzug von einer Verwaltungsbehörde an ein ordentli-

\footnotetext{
${ }^{1}$ ErläutRV 1618 BlgNR 24. GP zu Z 43.

2 Erstmals in VfGH G 13/54, VfSlg 2778/1954.

${ }^{3}$ Entwickelt in VfGH G 13/55, 18/55 VfSlg 2902/1955.
} 
ches Gericht für verfassungswidrig erachtet hatte, von den Absichten des historischen Verfassungsgesetzgebers umfasst gewesen war. Gegen diese Annahme sind zuletzt von Ewald Wiederin überzeugende Bedenken geäußert worden, ${ }^{4}$ die hier mit den Methoden der Rechtsund Verfassungsgeschichte kritisch nachvollzogen werden sollen. Denn wenn Wiederins Beobachtung stimmt, dass ein solches Verständnis des Grundsatzes ursprünglich nicht intendiert war, sondern dieses vielmehr ein Produkt der Verfassungsrechtsprechung der Nachkriegszeit ist, stellt sich unmittelbar die Frage, wie gewaltenübergreifende Instanzenzüge in den ersten Jahrzehnten des B-VG von den Richtern des VfGH beurteilt wurden.

\section{Art. 94 in der B-VG-Stammfassung}

\section{A. Zur Entstehungsgeschichte des Art. 94}

Art. 94 umfasste bis zur Verfassungsnovelle von 1929 drei Absätze. Abs. 1 ist stets unverändert geblieben und lautete von Anfang an: „Die Justiz ist von der Verwaltung in allen Instanzen getrennt.“ Abs. 2 lautete: „Wenn eine Verwaltungsbehörde über Privatrechtsansprüche zu entscheiden hat, steht es dem durch diese Entscheidung Benachteiligten frei, falls nicht im Gesetz anderes bestimmt ist, Abhilfe gegen die andere Partei im ordentlichen Rechtsweg zu suchen." Sowohl Abs. 1 als auch Abs. 2 haben ihre historischen Vorläufer. Abs. 1 entspricht mit einer geringfügigen Modifizierung dem Art. 14 StGG-RiG 1867; Abs. 2 kommt aus Art. 15 Abs. 1 StGG-RiG in das B-VG. Bereits hier lässt sich

\footnotetext{
${ }^{4}$ WIEDERIN, Instanzen 44ff. mit zahlreichen Nachweisen zur herrschenden Auslegung (vor 2014): Anm. 13 (Lehrbücher), Anm. 14 (Kommentare), Anm. 15 (Einführungswerke).
}

eine erste Beobachtung festhalten: Art. 94 enthält Absätze aus ursprünglich separaten Verfassungsartikeln, die erst 1920 in ihre neue Ordnung zusammengeführt wurden. Abs. 3 schließlich enthielt eine Bestimmung über die Zuständigkeit in Fragen der Bodenreform, die für die hier interessierende Problematik ohne Bedeutung ist und daher in der Folge ausgeklammert werden kann. ${ }^{5}$

In einem ersten Schritt soll nun untersucht werden, ob aus Art. 94 Abs. 2 idF BGBl 1/1920 sachdienliche Hinweise zur Zulässigkeit oder Unzulässigkeit gewaltenübergreifender Instanzenzüge zu gewinnen sind. Ist diese Frage geklärt, soll im Anschluss dasselbe mit dem bis heute in Kraft befindlichen Abs. 1 versucht werden.

\section{B. Art. 94 Abs. 2 hinsichtlich des Problems der Instanzenzüge}

Wie eben bemerkt, findet sich die Wurzel des Abs. 2 in Art. 15 Abs. 1 StGG-RiG. Diese Bestimmung lautete: „In allen Fällen, wo eine Verwaltungsbehörde nach den bestehenden oder künftig zu erlassenden Gesetzen über einander widerstreitende Ansprüche von Privatpersonen zu entscheiden hat, steht es dem durch diese Entscheidung in seinen Privatrechten Benachtheiligten frei, Abhilfe gegen die andere Partei im ordentlichen Rechtswege zu suchen." Diesem Satz folgte als Abs. 2 eine Bestimmung, die 1920 keine Aufnahme in das B-VG gefunden hat: „Wenn außerdem Jemand behauptet, durch eine Entscheidung oder Verfügung einer Verwaltungsbehörde in seinen Rechten verletzt zu sein, so steht ihm frei, seine Ansprüche vor dem Verwaltungs-Gerichtshofe im öffentlichen

\footnotetext{
5 „In den Angelegenheiten der Bodenreform (Artikel 12, Absatz 1, Zahl 6) steht den aus Richtern, Verwaltungsbeamten und Sachverständigen bestehenden Kommissionen das ausschließliche Entscheidungsrecht zu."
} 
mündlichen Verfahren wider einen Vertreter der Verwaltungsbehörde geltend zu machen." ${ }^{\text {"6 }}$

Während der nicht in das B-VG übernommene Abs. 2 ohne Zweifel einen Instanzenzug anspricht, nämlich einen solchen von einer Verwaltungsbehörde an den $\mathrm{VwGH}$, ist dies bei Abs. 1 augenscheinlich nicht der Fall. Dort wird nämlich Abhilfe nicht gegen eine Verwaltungsbehörde bzw. deren Entscheidung angestrebt, sondern gegen "die andere Partei“, also jene Privatperson $B$, gegenüber welcher der unterlegene Private $\mathrm{A}$ aufgrund einer verwaltungsbehördlichen Entscheidung über einander widerstreitender Ansprüche von A und B, welche privatrechtlicher Natur sind, in den Nachteil gesetzt wurde. Ohne rechtshistorische Detailkenntnisse lässt sich aus dieser Feststellung allein noch nichts gewinnen.

Die österreichische Rechtsordnung des 19. Jahrhunderts kannte vereinzelt Konstellationen, in denen rein privatrechtliche Konflikte nicht vor den ordentlichen Gerichten, sondern vor Verwaltungsbehörden auszutragen waren. Ein illustratives Beispiel dafür sind Lohnstreitigkeiten zwischen Arbeitgebern und Arbeitnehmern: Vor den "politischen“ (also: Verwaltungs-)Behörden waren nach den Hofdekreten vom 18. April $1828^{7}$ und 21. November $1846^{8}$ Dienststreitigkeiten zwischen Dienstgebern und Dienstboten, Gewerbsleuten oder Fabriksinha-

\footnotetext{
${ }^{6}$ Diese beiden Absätze und ihr Verhältnis zueinander bereiteten schon den Zeitgenossen größte Probleme bei der Auslegung, die insb. auch nicht durch die Heranziehung der Materialien gelöst werden konnten; vgl. unter vielen BERNATZIK, Rechtsprechung 247ff. Plausiblerweise deutet „außerdem“ in Abs. 2 darauf hin, dass die Beschwerde vor dem VwGH gegenüber der gerichtlichen Konfliktlösung nach Abs. 1 als subsidiär gedacht war, insofern als Genugtuung für das verletzte Recht zunächst von einem andernfalls unrechtmäßig bereicherten Privaten $\mathrm{zu}$ fordern und nur bei Fehlen eines solchen der VwGH anzurufen war.

7 JGS 2340.

8 JGS 1002
}

bern und deren Gesellen, Lehrjungen oder anderen Hilfsarbeitern abzuhandeln, welche aus dem Dienstverhältnis oder Lohnvertrag hergeleitet und während des Bestands des Dienstverhältnisses oder wenigstens vor Ablauf von $30 \mathrm{Ta}-$ gen, vom Ende des Dienstvertrages an gerechnet, „angebracht" wurden. Nur solche Forderungen, die erst nach Ablauf dieser Frist erhoben wurden, waren vor den ordentlichen Gerichten geltend zu machen. ${ }^{9}$

Gegen die wirtschaftlichen Folgen einer solchen Entscheidung einer Verwaltungsbehörde, nicht aber gegen das Bestehen der Entscheidung selbst, konnte nun nach Art. 15 Abs. 1 StGG-RiG vor dem ordentlichen Gericht Abhilfe begehrt werden. ${ }^{10}$ Die Funktion dieser Norm lag also darin, klarzustellen, dass die verwaltungsbehördliche Entscheidung keinen präjudiziellen Charakter hinsichtlich der widerstreitenden Ansprüche der Parteien insgesamt entfaltete, sondern die Entscheidung der Verwaltungsbehörde auf den Gegenstand der ihr die Kompetenz verschaffenden Norm, im Beispielsfall also Ansprüche, die unmittelbar aus dem Dienstvertrag abgeleitet wurden, beschränkt war. ${ }^{11}$

\footnotetext{
${ }^{9} \mathrm{Vgl}$. außer den genannten Fundstellen auch RGBl. 224/1856 und 73/1860, sowie ähnlich § 102 GewO 1856, RGBl. 227.

${ }^{10}$ So auch die Materialien: „[...] so muss dem Benachtheiligten der ordentliche Rechtsweg gegen die andere Partei offen stehen, um auf diesem Wege die nachteiligen Folgen dieser Entscheidung beseitigen oder die Entschädigung erlangen zu können“, Motivenbericht des Verfassungsausschusses des Abgeordnetenhauses, zitiert nach BERNATZIK, Rechtsprechung 248.

${ }^{11}$ Vgl. auch N. N. [SAMITSCH], Correctur 8: „Wäre z.B. der Arbeitgeber durch die verwaltungsbehördliche Entscheidung zur Zahlung eines Arbeitslohnes an den Arbeiter verhalten worden, so kann durch den civilgerichtlichen Ausspruch die Rückerstattung desselben an den Arbeitgeber verfügt werden, wenn z.B. erkannt wird, daß dem letzteren ein eben so hoher Betrag wider den Arbeiter etwa aus dem Titel des Schadenersatzes gebühre, daher die Compensation eintrete."
} 
Im Herbst 1918 trat nach der deutschösterreichischen Staatsgründung an die Stelle des StGGRiG das GGrG vom 22. November 1918. ${ }^{12}$ Die hier besprochene Bestimmung wurde als nunmehriger $\S 18$ Abs. 1 nahezu gleichlautend übernommen. ${ }^{13} 1920$ erfolgte die Rezeption in das B-VG, wobei belegt ist, dass sich Hans Kelsen explizit dagegen ausgesprochen hatte. ${ }^{14}$

In der Fassung von 1920 erhielt der nunmehrige Art. 94 Abs. 2 einen Gesetzesvorbehalt, außerdem wurde der Verweis auf „bestehende oder künftig zu erlassende Gesetzen" gestrichen: „Wenn eine Verwaltungsbehörde über Privatrechtsansprüche $\mathrm{zu}$ entscheiden hat, steht es dem durch diese Entscheidung Benachteiligten frei, falls nicht im Gesetz anderes bestimmt ist, Abhilfe gegen die andere Partei im ordentlichen Rechtsweg zu suchen." Die normative Aussage war trotz kleinerer Änderungen im Lauf der inzwischen vergangenen dreiundfünfzig Jahren die gleiche geblieben. Über das Problem eines gewaltenübergreifenden Instanzenzuges trifft Art. 94 Abs. 2 idF BGBl 1/1920 keine Aussage. ${ }^{15}$ Für alle weiterführenden Überlegungen dazu ist er also unbeachtlich.

\footnotetext{
12 StGBl. 38/1918; BRAUNEDER, Verfassungsgeschichte 197.

${ }^{13} \S 18$ Abs. 1 GGrG 1918 lautete: „In allen Fällen, wo eine Verwaltungsbehörde nach den bestehenden oder künftig zu erlassenden Gesetzen über Privatrechtsansprüche zu entscheiden hat, steht es dem durch diese Entscheidung Benachteiligten frei, Abhilfe gegen die andere Partei im Rechtsweg zu suchen."

${ }^{14}$ Kelsen trat zuletzt für die vollständige Streichung von Art. 94 ein; vgl. WALTER, Entstehung 196.

${ }^{15}$ Anders die ErläutRV 1618 BlgNR 24. GP 11, die Art. 94 Abs. 2 idF BGBl 1/1920 offenbar als Ermächtigung zur Schaffung von Ausnahmen vom Grundsatz der Trennung von Justiz und Verwaltung verstanden wissen wollen.
}

\section{Art. 94 Abs. 1 hinsichtlich des Problems der Instanzenzüge}

Ungleich weniger kompliziert gestaltet sich die Auslegung von Art. 94 Abs. 1. Seinen Ursprung hat er in Art. 14 StGG-RiG: „Die Rechtspflege wird von der Verwaltung in allen Instanzen getrennt." Emmerich Coreth zufolge ist dieser Satz ,zunächst sicherlich nur als eine erneute Bekräftigung des Bekenntnisses zu jener theoretischen Lehrmeinung von der Gewaltenteilung zu werten", wonach die Regierungsgewalt, die der Kaiser „durch verantwortliche Minister und die denselben untergeordneten Beamten und Bestellten ausübt" (RGBl 145/1867), von der richterlichen Gewalt, die zwar im Namen des Kaisers, aber durch unabhängige Richter ausgeübt wird, verschieden ist. ${ }^{16}$ Eine Abgrenzung der verschiedenen Materien, die zu den Aufgaben des Staates zählen, hinsichtlich ihrer Zuordnung zu entweder "Rechtspflege“ oder Verwaltung ist Art. 14 nicht zu entnehmen. Der normative Gehalt der Bestimmung ist vielmehr ein organisatorischer: Neben den Verwaltungsbehörden hatten in den im Reichsrat vertretenen Königreichen und Ländern auch Gerichte zu bestehen, deren Unabhängigkeit im Sinne des StGG-RiG gewährleistet war. Diese beiden „Behördengattungen" waren zudem in organisatorischer Hinsicht so voneinander $\mathrm{zu}$ scheiden, dass keine Behörde jemals gleichzeitig Verwaltungsbehörde und Gericht sein konnte. Hat man diesen Regelungsinhalt einmal festgestellt, ist auch die eigentümliche Formulierung des Satzes (,wird [...] in allen Instanzen getrennt ${ }^{\prime \prime}$ ) leicht erklärt: Regelungszweck war die Abschaffung und das in die Zukunft gerichtete Verbot der sogenannten "Gemischten Bezirksämter", die als Relikte des Neoabsolutismus fortbestanden und neben der Funktion der Bezirksverwaltungsbe-

${ }^{16}$ CORETH, Anfechtung 2, insb. auch zur Problematik der verwendeten Terminologie. 
hörde auch jene des Bezirksgerichts ausübten. ${ }^{17}$ Deshalb wird auch die Trennung von „Rechtspflege" und Verwaltung (noch) nicht als bestehende Tatsache postuliert, sondern als „Forderung und Befehl für die Zukunft" formuliert; die ausdrückliche Nennung „aller Instanzen“ verweist also auf die erste, unterste Instanz, denn nur auf dieser Ebene war die Trennung noch zu vollziehen. ${ }^{18}$ Die Ausführung der in Art. 14 enthaltenen Anordnung erfolgte sodann mit dem Gesetz über die Einrichtung der politischen Landesbehörden vom 19. Mai 1868. ${ }^{19}$

Das deutschösterreichische GGrG übernahm den Wortlaut des Art. 14 StGG-RiG in seinen $\S 17$, ergänzt um den Satz: „Die Staatsanwaltschaft gilt als Verwaltungsbehörde." Diese Ergänzung nahm der Verfassungsgesetzgeber des Jahres 1920 wieder zurück, ersetzte den unklaren Begriff "Rechtspflege“ durch "Justiz" und tauschte, da die Gemischten Bezirksämter längst der Vergangenheit angehörten, das anachronistische Futurum gegen ein angemesseneres Präsens: „Die Justiz ist von der Verwaltung in allen Instanzen getrennt."

Ein Verbot eines gewaltenübergreifenden Instanzenzuges von einer Verwaltungsbehörde zu einem ordentlichen Gericht, oder auch umgekehrt, lässt sich weder aus dem Wortlaut von Art. 94 Abs. 1 noch aus dessen Vorläuferbestimmungen ableiten. Zudem enthielt zunächst das VwGHG 1876 in § 3 lit. a, später Art. 131 Z 2 B-VG idF BGBl. 1/1920 (bis zur Verfassungsnovelle 1929) eine Bestimmung, der zufolge Angelegenheiten, über welche den ordentlichen Gerichten die Entscheidung zustand, von der $\mathrm{Zu}$ ständigkeit des VwGH ausgenommen waren. Dies wurde damit begründet, dass der Zweck der Verwaltungsgerichtsbarkeit, nämlich die gerichtliche Kontrolle der Verwaltung, durch

\footnotetext{
${ }^{17} \mathrm{Zu}$ den „Gemischten Bezirksämtern“ vgl. BrauNEDER, Verfassungsgeschichte 146, 174.

${ }^{18}$ CORETH, Anfechtung 3f.

${ }^{19}$ RGB1. 44/1868.
}

die Befassung der ordentlichen Gerichtsbarkeit eben schon erfüllt sei. ${ }^{20}$

Als Zwischenergebnis ist also festzuhalten, dass zumindest der Stammfassung des B-VG 1920 ein Verbot gewaltenübergreifender Instanzenzüge fremd zu sein scheint. Wenn diese These stimmt, liegt die Vermutung nahe, dass derartige Rechtszüge tatsächlich stattgefunden und ihre Spuren hinterlassen haben - optimaler Weise in der Rechtsprechung des VfGH. Eine solche Spur soll im nächsten Abschnitt dieses Beitrags verfolgt werden.

\section{Der Instanzenzug im Vorkriegsschuldengesetz}

\section{A. Die Vorkriegsschuldenkrise und ihre rechtlichen Folgen}

Der Erste Weltkrieg war nicht zuletzt ein globaler Wirtschaftskrieg. Entsprechend einschneidend waren die Konsequenzen der Handelsund Zahlungsverbote, die nach Ausbruch des Krieges gegenüber den jeweiligen Feindstaaten verhängt wurden. Diese verfolgten einerseits den Zweck, die gegnerischen Volkswirtschaften durch die Blockierung der Geldflüsse ins Land zu schwächen, dienten andererseits aber auch dazu, Verfügungsgewalt über die Forderungen feindlicher Staatsangehöriger zu erlangen, um diese bei späteren Friedensverhandlungen als Druckmittel einsetzen zu können. Gemeinsam ist diesen Instrumenten, dass sie in privatrechtliche Verhältnisse eingriffen und dazu führten, dass bereits bestehende Verbindlichkeiten auf unbestimmte Zeit nicht abgewickelt werden konnten. Schon während des Krieges war den beteiligten Staaten klar, dass mit der Wiederaufnahme friedlicher Wirtschaftsbeziehungen alle diese Verbindlichkeiten in ein und demselben

${ }^{20}$ Kelsen, Froehlich, MerKL, Bundesverfassung $244 \mathrm{f}$. 
Moment reaktiviert werden würden, was zwingend die Tragfähigkeit der Märkte überlastet und zu einem "ökonomischen Kurzschluss" geführt hätte.

Dem Kriegsausgang entsprechend, bestimmten die Vorstellungen der Alliierten die in den Pariser Vororteverträgen enthaltenen privatrechtlichen Bestimmungen. Der für Österreich maßgebliche Vertrag von Saint-Germain-en-Laye regelte die Behandlung der sogenannten „Vorkriegsschulden" in Art. 248f. samt Anhängen. Nach dem dort vorgesehenen Abrechnungsverfahren waren die vom Clearingregime erfassten Geldverbindlichkeiten über Vermittlung sogenannter „Prüfungs- und Ausgleichsämter", die von jeder der teilnehmenden Vertragsparteien zwingend einzurichten waren, auf zwischenstaatlicher Ebene abzuwickeln. Jeglicher direkter Verkehr zwischen Schuldnern und Gläubigern war durch die Vertragsstaaten zu unterbinden und es hafteten diese, von wenigen Ausnahmefällen abgesehen, für die Bezahlung der Schulden ihrer jeweiligen Staatsangehörigen. ${ }^{21}$

Die Umsetzung dieser völkerrechtlichen Vorgaben in das innerstaatliche Recht Österreichs erfolgte durch das Vorkriegsschuldengesetz. ${ }^{22}$ Neben den größtenteils völkerrechtlich vorgegebenen Maßnahmen zur Durchführung des sogenannten „äußeren Clearings“, also der zwischenstaatlichen Abwicklung der Verbindlichkeiten, war auch die Ausgestaltung des ,inneren Clearings" zu regeln, worunter das Verhältnis zwischen dem (haftenden) Vertragsstaat und den ihm zurechenbaren privaten Gläubigern bzw. Schuldnern verstanden wurde. Das zentrale Problem war hierbei das Ausmaß des „Schuldenschnitts", der vor dem Hintergrund der negativen Entwicklung der Kronenwährung sowie der Verpflichtung, das „äußere Clearing“ in der Währung des beteiligten Siegerstaates durchzu-

\footnotetext{
${ }^{21}$ Art. 248 StV von Saint-Germain, StGBl. 303/1920.

22 BGBl. 393/1921
}

führen, zur Entlastung der Wirtschaftstreibenden in das Paket aufgenommen werden sollte. Zum nationalen Prüfungs- und Ausgleichsamt wurde das Abrechnungsamt in Wien bestimmt, dem die Abwicklung sowohl des „inneren“ als auch des „„äußeren Clearings“ oblag (§ 2 Abs. 1).

Das Abrechnungsamt war ein öffentliches, dem Staatsamt für Finanzen unterstehendes Amt, dessen Aufgaben und Wirkungskreis durch eine von diesem im Verordnungsweg erlassene Satzung geregelt wurde. Nach dieser Satzung oblag die Leitung des Abrechnungsamtes dessen Direktorium, bestehend aus einem Präsidenten, zwei Vizepräsidenten, drei Staatskommissären und höchstens drei leitenden Beamten. Der Präsident, die beiden Vizepräsidenten und die Staatskommissäre wurden vom Staatssekretär für Finanzen auf die Dauer eines Jahres ernannt. Diese sechs Funktionäre beriefen die dem Direktorium angehörenden leitenden Beamten. Der Staatssekretär für Finanzen konnte die von ihm ernannten Personen jederzeit ihrer Ämter entheben. ${ }^{23}$ Aus diesen Umständen ergibt sich zweifelsfrei, dass das Abrechnungsamt nicht der Staatsfunktion Justiz, sondern der Verwaltung zuzurechnen war.

Gegen Abrechnungen und sonstige Entscheidungen des Abrechnungsamtes (von einzelnen, im Gesetz angeführten, Ausnahmen abgesehen) konnte innerhalb von 30 Tagen die Vorstellung an das Abrechnungsamt ergriffen werden. Gegen eine Entscheidung wiederum, mit welcher eine Vorstellung ganz oder teilweise abgewiesen worden war, konnte binnen 30 Tagen im „ordentlichen Rechtswege" das Rechtsmittel der Beschwerde an den Abrechnungsgerichtshof ergriffen werden, wenn der Beschwerdeführer behauptete, durch eine rechtswidrige Entscheidung des Abrechnungsamtes in seinen Rechten verletzt worden $\mathrm{zu}$ sein (§55 Abs.1-3). Der

\footnotetext{
${ }^{23}$ Vollzugsanweisung des Staatsamtes für Finanzen vom 9.1.1920 über Errichtung und Aufgaben des Abrechnungsamtes, StGBl. 25.
} 
Abrechnungsgerichtshof war außerdem hinsichtlich im Inland zu erfüllender Valutaverbindlichkeiten zwischen Privaten zuständig für die Entscheidung über Parteienanträge auf Stundung bestimmter Schulden, Nachsicht einer nach dem Gesetz zu leistenden Sicherheit oder Abänderung eines bestehenden Lebensversicherungsvertrags ( $\S \S 48 \mathrm{ff}$.). Hinsichtlich seiner Kompetenzen übte der Abrechnungsgerichtshof also sowohl verwaltungs- als auch zivilgerichtliche Funktionen aus.

Der Abrechnungsgerichtshof war nach § 56 als „ordentliches Gericht" eingerichtet und für die Rechtsmittel nach $\S 55$ ausschließlich zuständig. Warum der einfache Bundesgesetzgeber der Ansicht war, in diesem Fall einen Instanzenzug von einer Verwaltungsbehörde an ein ordentliches Gericht vorsehen zu müssen, geht aus den Materialien sehr instruktiv hervor: Der VwGH und der VfGH erschienen für diese Aufgabe nicht geeignet, da sie „nach ihrer Tätigkeit den auf Grund des Gesetzes zu behandelnden Fragen ferne" stünden und "ohne eine sehr bedeutende Änderung der Organisation und Vermehrung des Personalstandes die durch das [...] Gesetz erwachsenden Aufgaben nicht bewältigen“ könnten: „Wird es sich doch in vielen Fällen um ein Verfahren handeln, das am ehesten einem Rechnungsprozeß nach der Zivilprozeßordnung gleichzustellen ist, in der [sic!] umständliche Prüfungen von Bilanzen, kaufmännischen Korrespondenzen und dergleichen vorzunehmen sein werden. Auch erscheint es notwendig, auch Fachkreise heranzuziehen, was nach der Organisation des Verfassungsgerichtshofes und des Verwaltungsgerichtshofes nicht möglich wäre." Aus ähnlichen Zweckmäßigkeits- und Effizienzüberlegungen wurde die Option eines Instanzenzuges an die bestehenden ordentlichen Gerichte ebenfalls verworfen, so- dass die Errichtung eines neuen Gerichtshofes als einzige verbleibende Alternative erschien. ${ }^{24}$

Das Verfahrensrecht des Abrechnungsgerichtshofs war im Wesentlichen jenem des Verwaltungsgerichtshofs nachgebildet. Die Verhandlungen waren in der Regel öffentlich zu führen, wohingegen die Beratung und Abstimmung über das Urteil nicht öffentlich zu erfolgen hatten. Dieses sollte nach Möglichkeit sogleich nach Schluss der mündlichen Verhandlung gefällt und mit den wesentlichen Entscheidungsgründen verkündet werden. Das Urteil war binnen acht Tagen nach Schluss der Verhandlung schriftlich auszufertigen und begründete einen Exekutionstitel. ${ }^{25}$

\section{B. Die Besetzung des Abrechnungsgerichtshofs}

Die Entstehungsgeschichte des Gerichtshofs reflektiert die Bedeutung, die den involvierten Partikularinteressen zukam. Nach einem frühen Departementsentwurf aus dem November 1920 hätte der Abrechnungsgerichtshof aus einem Präsidenten, vier Räten und vier Ersatzmännern bestehen sollen. Während der Präsident vom Bundespräsidenten zu ernennen gewesen wäre, wären die Räte und Ersatzmänner aufgrund einer Ermächtigung des Bundespräsidenten vom Bundesminister für Finanzen jeweils für ein Jahr, jedoch mit der Möglichkeit einer Wiederbestellung, zu ernennen gewesen. Unbeachtlich dieser Befristung sollte die Unabhängigkeit der Mitglieder in der Ausübung ihres richterlichen Amtes nach dem Wortlaut des Gesetzesentwurfs garantiert sein. ${ }^{26}$

Bereits wenige Monate später, nämlich Ende Februar 1921, lag ein weiterer Entwurf vor, der eine den Handelsgerichten nachgebildete Orga-

\footnotetext{
${ }^{24}$ ErläutRV 395 BlgNR 1. GP 48f.

${ }^{25}$ Verordnung vom 20.7.1922 über die Verfassung und das Verfahren des Abrechnungsgerichtshofes, BGB1. 562, §§ 14ff.

${ }^{26}$ Departementsentwurf 23. 11. 1920, §§ 39ff.
} 
nisation des Abrechnungsgerichtshofs vorsah: Dieser sollte nunmehr in Senaten entscheiden, die aus einem Vorsitzenden und vier Beisitzern zu bestehen hatten. Von den Beisitzern hatte einer jenen Kreisen anzugehören, aus denen die fachmännischen Laienrichter bei den Handelsgerichten bzw. den Handelssenaten der Gerichtshöfe erster Instanz $\mathrm{zu}$ entnehmen waren. Die Ernennung des Präsidenten sowie der Mitglieder des Abrechnungsgerichtshofs sollte auch hier durch den Bundespräsidenten auf Vorschlag der Bundesregierung erfolgen, welcher wiederum auf einvernehmlichen Antrag der Bundesminister für Finanzen und für Justiz zu erstatten gewesen wäre. Eine Befristung der Ernennung war nicht mehr vorgesehen. ${ }^{27}$

Der Übergang von dieser zunächst viertelparitätischen Besetzung zu einer weitergehenden Einbeziehung der Wirtschaftstreibenden erfolgte im Mai 1921 mit einem neuen Departementsentwurf, nach dem sogar die Hälfte der Mitglieder des Gerichtshofs aus den genannten Kreisen rekrutiert werden sollte. Vorschlagen sollte diese Laienrichter der Handelskammertag (bestehend aus den Präsidenten und Vizepräsidenten der nach dem Gesetz vom 25. Februar 1920 über Kammern für Handel, Gewerbe und Industrie eingerichteten Kammern und den Direktoren der jeweiligen Kammerämter ${ }^{28}$ ). Dementsprechend war auch für die Senate vorgesehen, dass zwei der vier Beisitzer aus Laienkreisen zu stammen hätten. ${ }^{29}$ Diese Aufwertung der Laienbeteiligung ist im Zusammenhang mit dem hohen Stellenwert zu sehen, den die Interessenpolitik der Wirtschaftstreibenden schon bei der Administration der Handels- und Zahlungsverbote während des Weltkriegs gehabt hatte. So beruhte insbesondere die Organisation des Abrechnungsamts auf jener seiner Vorläuferinstitu-

\footnotetext{
${ }^{27}$ Departementsentwurf 26. 2. 1921, § 37.

${ }^{28}$ Gesetz vom 25. 2. 1920 über Kammern für Handel, Gewerbe und Industrie, BGBl. 98, §§ 31 iVm 14, 27.

${ }^{29}$ Departementsentwurf 28. 5. 1921, § 56.
}

tion, der von den Handels- und Gewerbekammern und dem Handelsmuseum gemeinsam eingerichteten „Schutzstelle für (deutsch)österreichische Vermögen im Auslande“, die im Herbst 1917 per Verordnung mit Rechtspersönlichkeit versehen und in Umsetzung des Staatsvertrags von Saint-Germain Anfang 1920 in ein staatliches Amt, eben das Abrechnungsamt, umgewandelt worden war. ${ }^{30}$

Dem zuletzt erörterten Entwurf folgte dann auch die im Nationalrat beschlossene Fassung des VKSchG. Die Senate bestanden somit neben dem Vorsitzenden jeweils aus zwei laienrichterlichen und zwei rechtskundigen Mitgliedern, wobei einer dieser Beisitzer Richter von Beruf sein musste (§ 56). Hinsichtlich der Zusammensetzung des Gerichtshofs wurde später im Verordnungsweg ergänzt, dass dieser neben dem Präsidenten und dem Vizepräsidenten 24 weitere Mitglieder umfassen sollte, wobei die Zahl der Mitglieder einer Erhöhung wiederum mittels Verordnung offenstand. ${ }^{31}$

\section{Verfassungsrechtliche Probleme im Vorfeld}

\section{Offene Rechtsfragen}

Während der von den Bundesministerien für Justiz und für Finanzen einvernehmlich zu machende Vorschlag hinsichtlich der Richter des Abrechnungsgerichtshofs erarbeitet wurde, wurde offenkundig, dass zwei verfassungsrechtliche Probleme bis dahin nicht bedacht worden waren. Zum einen enthielt die im Justizministe-

\footnotetext{
${ }^{30}$ Verordnung des Handelsministers im Einvernehmen mit den beteiligten Ministern vom 10.10.1917 über die Errichtung einer Schutzstelle für österreichische Vermögen im Auslande, RGBl. 404; Vollzugsanweisung des Staatsamtes für Finanzen vom 9.1.1920 über Errichtung und Aufgaben des Abrechnungsamtes, StGB1. 25.

${ }^{31}$ Verordnung vom 20.7.1922 über die Verfassung und das Verfahren des Abrechnungsgerichtshofes, BGB1. 562, § 1 .
} 
rium ausgearbeitete Liste, die von dort dem Finanzministerium mit dem Ersuchen um $\mathrm{Zu}$ stimmung zur Kenntnis gebracht wurde, die Namen dreier hochrangiger Beamter des eigenen Hauses, was im Finanzressort Bedenken hervorrief: Ein Richter, der im Hauptberuf aktiver Verwaltungsbeamter sei, könne nicht als unabhängig und unabsetzbar im Sinne des Art. 87 B-VG angesehen werden. Da zudem das Justizministerium die administrative Aufsicht über den Gerichtshof habe, könne nicht ausgeschlossen werden, dass die betreffenden Beamten in der Lage wären, das Verhalten des Ministeriums gegenüber dem Gerichtshof zu beeinflussen. Somit bestünde die Gefahr, dass die Öffentlichkeit diese Richter vornehmlich als Verwaltungsbeamte, die den Weisungen des Ministers unterstünden, wahrnehmen könnte. Zum anderen wurde moniert, dass vom Justizministerium lediglich zwölf vom Handelskammertag namhaft gemachte potentielle Laienrichter mitgeteilt worden waren - also nur so viele, als Stellen zu besetzen waren -, sodass dem Finanzministerium keine Möglichkeit blieb, allenfalls einzelne Kandidaten als ungeeignet abzulehnen. Da der dem Ministerrat vorzulegende Besetzungsvorschlag mindestens doppelt so viele Personen zu umfassen habe, als Richter zu ernennen seien, müssten nach Ansicht des Finanzministeriums schon allein deshalb zumindest 24 geeignete Laien namhaft gemacht werden. Dabei berief man sich auf Art. 86 Abs. 2 B-VG. Dieser lautet: „Der dem zuständigen Bundesminister vorzulegende und der von ihm an die Bundesregierung zu leitende Besetzungsvorschlag hat, wenn genügend Bewerber vorhanden sind, mindestens drei Personen, wenn aber mehr als eine Stelle zu besetzen ist, mindestens doppelt so viele Personen zu umfassen, als Richter zu ernennen sind." Solche Besetzungsvorschläge waren nach Abs. 1 desselben Artikels von den "durch die Gerichtsverfassung hiezu berufenen Senaten" zu erstatten.
Zur Klärung dieser verfassungsrechtlichen Fragen wurde ein Gutachten des bekannten Staatsrechtslehrers Hans Kelsen eingeholt.

\section{Das Gutachten Hans Kelsens}

Aus Kelsens Sicht war zunächst zu klären, wie der Trennungsgrundsatz des Art. 94 Abs. 1 BVG richtigerweise auszulegen sei. Dazu setzte er bei der historischen Ausgangssituation an: Bereits in vorkonstitutioneller Zeit habe man begrifflich zwischen Justiz und Verwaltung unterschieden, ohne jedoch zu fordern, dass die Ausübung der beiden Funktion nicht in einer Person vereinigt sein dürfe. „Wenn diesem Zustand gegenüber postuliert wurde, Justiz und Verwaltung zu trennen, so konnte die Forderung wohl kaum einen anderen Sinn haben", so Kelsen, „als die Justiz- und Verwaltungsfunktion auf verschiedene Organträger, d.h. auf verschiedene Menschen, als Funktionäre zu übertragen“. Hinter diesem Schritt sei weniger das Bedürfnis, den Richtern die Unabhängigkeit und Unversetzbarkeit zu gewähren, gestanden, als vielmehr die Überlegung, dass Verwaltungsbeamte gewohnt seien, sich „innerhalb des Rahmens freien Ermessens, das die Verwaltungsrechtsnormen einräumen" viel mehr am Grundsatz der Zweckmäßigkeit „als an dem der formalen Rechtmäßigkeit zu orientieren“. Diese seien daher aufgrund ihrer Arbeitsweise nicht geeignet, die Justizgesetze zu vollziehen, wo es umgekehrt darauf ankomme, "vor allem die formale Rechtmässigkeit und erst in zweiter Linie, soweit dies überhaupt in dem engen Rahmen der Justizgesetze möglich ist, die Zweckmässigkeit zu beachten“. Die Forderung, Justiz und Verwaltung zu trennen, bedeute daher in erster Linie, dass die Personen, denen die Ausübung von Justiz und Verwaltung obliegt, verschieden zu sein hätten.

Jedoch, räumte Kelsen ein, sei die „Erfüllung dieser Forderung verschiedener Grade fähig“, ihre Reichweite also nicht eindeutig bestimmt. Insbesondere sei die Mitgliedschaft in Kollegial- 
behörden, die der jeweils anderen Staatsfunktion zuzurechnen seien, als Grenzfall anzusehen. In diesem Zusammenhang erinnerte er daran, dass etwa aktive Verwaltungsbeamte als Mitglieder des VfGH tätig seien, ohne dass darin ein Widerspruch zu Art. 94 Abs. 1 B-VG gesehen würde. $\mathrm{Ob}$ diese Auffassung richtig sei, sei „eben eine Frage der Interpretation, die mit Rücksicht auf die mögliche Graduierung in der Erfüllung der Forderung des Art. 94 Abs. 1 kaum eindeutig erfolgen" könne. Einerseits müsste man streng genommen zu dem Ergebnis kommen, dass niemand, der in irgendeiner Form als Richter an einem staatlichen Gericht tätig sei, zugleich auch als Verwaltungsbeamter fungieren könne, und umgekehrt. Andererseits sei ein dermaßen strenges Verständnis des Trennungsgrundsatzes dem B-VG fremd, wo doch Art. 87 ausdrücklich zulasse, dass Richter auch als Organe der Justizverwaltung tätig werden könnten.

Hinsichtlich der konkreten Frage, ob ein Beamter des Justizministeriums zugleich auch die Funktion eines Richters des Abrechnungsgerichtshofs haben könne, vertrat Kelsen die Meinung, dass dies „selbst bei weniger rigoroser Interpretation eher" zu verneinen sei. Die im Finanzministerium gehegte Befürchtung, dass die Unabhängigkeit und Unabsetzbarkeit der Richter gefährdet sei, wenn sie im Stammberuf Verwaltungsjuristen des Justizministeriums wären, stand für ihn allerdings gegenüber dem formaljuristischen Argument im Hintergrund. Vollständig unerörtert aber blieb das hier in erster Linie interessierende Problem des Instanzenzugs von einer Verwaltungsbehörde an ein Gericht. Vielmehr: Für Kelsen stellte sich diese Frage gar nicht. ${ }^{32}$

Der zweite juristische Aspekt, dem sich Kelsens Gutachten zu widmen hatte, betraf die Anwendbarkeit des oben zitierten Art. 86 Abs. 2 B-

\footnotetext{
${ }^{32}$ Gutachten Kelsen 1ff.
}

VG auf die Bestellung der Richter des Abrechungsgerichtshofs. Fraglich war in diesem Zusammenhang, wie sich diese Verfassungsnorm $\mathrm{zu}$ den einfachgesetzlichen Bestimmungen des $\S 56$ Abs 1 u. 2 VKSchG verhielt. Wie bereits ausgeführt, waren demnach der Präsident, der Vizepräsident und die Mitglieder des Gerichtshofs über Vorschlag der Bundesregierung, der auf einvernehmlichen Antrag der Bundesminister für Finanzen und für Justiz zu erstatten war, vom Bundespräsident zu ernennen, wobei die Laienrichter den Ministerien vom Handelskammertag vorzuschlagen waren.

Dazu stellte Kelsen zunächst fest, dass als „Senate" im Sinne des Art. 86 Abs. 1 B-VG lediglich die Personalsenate der Gerichte selbst angesehen werden könnten. Daher sei es nicht möglich, "sich bei der Frage der Besetzungsvorschläge auf den Standpunkt der Verfassung zu stellen“. Möglich wäre eine verfassungskonforme Vorgehensweise daher erst nach erfolgter Konstituierung des Gerichtshofs und der darauf folgenden Bildung eines Personalsenats. Im Ergebnis sei daher die Frage, „ob und wer Besetzungsvorschläge an die zuständigen Minister zu erstatten habe, und wie diese Besetzungsvorschläge beschaffen sein müssen" nur auf der Grundlage des Vorkriegsschuldengesetzes beantwortbar. Mangels weiter gehender gesetzlicher Vorgaben müsse der Vorschlag des Handelskammertags daher nur so viele Namen enthalten, als Laienrichter zu ernennen seien. Bezüglich des Präsidenten, des Vizepräsidenten und der rechtskundigen Mitglieder seien die zuständigen Minister in ihrer Auswahl völlig frei. Ein vorgelagertes Auswahlverfahren, so Kelsen, sei wohl wünschenswert, jedenfalls aber nicht vorgeschrieben. ${ }^{33}$

${ }^{33}$ Ebd. 4; vgl. auch Kelsen, Froehlich, MerKL, Bundesverfassung $192 \mathrm{ff}$. 


\section{Die Umsetzung der gewonnenen Erkenntnisse}

Erörtert wurden diese Fragen auch bei einem interministeriellen Treffen im März 1922. Dabei stellte sich heraus, dass das Justizministerium den Handelskammertag von Vornherein nur zur Nennung von zwölf Personen aufgefordert hatte. Wenngleich das Finanzministerium an seiner Einschätzung festhielt, dass eine umfangreichere Liste mit entsprechender Auswahlmöglichkeit zweckmäßiger gewesen wäre, musste man doch einsehen, dass aufgrund der fortgeschrittenen Zeit ein ergänzter Vorschlag nicht mehr eingeholt werden konnte. Auch hinsichtlich der Berufsrichter wurde ein Konsens darüber erzielt, dass mangels Anwendbarkeit des Art. 86 B-VG die Zahl der vorgeschlagenen Personen auf eine der zu vergebenden Stellen entsprechende beschränkt bleiben konnte. Von einem Auswahlverfahren beschloss man mit Verweis auf die damit verbundene Verzögerung bei der Errichtung des Gerichtshofs abzusehen. Hinsichtlich der für Richterstellen vorgesehen aktiven Beamten des Justizministeriums ließ sich selbiges davon überzeugen, diese Nominierungen zurückzuziehen und akzeptierte den Vorschlag des Finanzressorts, stattdessen Richter des Verwaltungsgerichtshofs in den Vorschlag aufzunehmen. ${ }^{34}$

In der Ministerratssitzung vom 28. August 1922 genehmigte die Bundesregierung den von den Bundesministern für Justiz und für Finanzen unterbreiteten Vorschlag. Als Präsident des neuen Gerichtshofs wurde Gustav Walker eingesetzt, Ministerialrat des Justizministeriums ${ }^{35}$ und als Professor an der Wiener Universität eine anerkannte Autorität auf dem Gebiet des inter-

\footnotetext{
${ }^{34}$ BMF Zl. 111866/21.

${ }^{35} \mathrm{Um}$ die angesprochene Unvereinbarkeit nach Art. 94 Abs. 1 B-VG auszuschließen, erklärte sich Gustav Walker bereit, im Falle seiner Ernennung als Ministerialbeamter in den Ruhestand zu treten: BMF Zl. 111866/21.
}

nationalen Privatrechts. Für das Amt des Vizepräsidenten war der Senatspräsident des Obersten Gerichtshofs Johann Fischböck vorgesehen. Die Liste der rechtskundigen Mitglieder umfasste je vier Richter des Obersten Gerichtshofs und des Verwaltungsgerichtshofs, zwei emeritierte Richter des Oberlandesgerichts Wien sowie je einen Richter des Wiener Landes- und Handelsgerichts. Von den zwölf vom Handelskammertag genannten Laienrichtern wurden im Ministerrat nur acht zum Vorschlag gebracht; die übrigen vier waren als nicht voll geeignet abgelehnt worden. Diesbezüglich hatte der Handelskammertag bei seinem nächsten Zusammentreten eine Nachnominierung vorzunehmen. ${ }^{36}$ Am 16. Oktober 1922 nahm der Abrechnungsgerichtshof schließlich seine Tätigkeit auf. ${ }^{37}$

\section{Der Instanzenzug nach dem VKSchG vor dem VfGH}

Mit dem Instanzenzug vom Abrechnungsamt an den Abrechnungsgerichtshof hatte sich der VfGH schon sehr bald $\mathrm{zu}$ befassen, nachdem eine Partei gemäß Art. 144 B-VG gegen eine Entscheidung des Abrechnungsgerichtshofs eine Beschwerde an den VfGH wegen einer behaupteten Verletzung verfassungsmäßig gewährleisteter Rechte, konkret des Rechts auf den gesetzlichen Richter, erhoben hatte.

Über diese Beschwerde beriet der VfGH an zwei Terminen im November und Dezember 1923 in nichtöffentlicher Sitzung. Referent Robert Neumann-Ettenreich vertrat die Auffassung, die Beschwerde wäre wegen Nichtzuständigkeit des VfGH zurückzuweisen, da der Abrechnungsge-

${ }^{36}$ MRP 220, 28. 8. 1922; Wiener Zeitung, Nr. 203 vom 16. 9. 1922, 1.

${ }^{37}$ Kundmachung des Bundesministeriums für Justiz im Einvernehmen mit dem Bundesministerium für Finanzen vom 2. 10. 1922 über den Beginn der Tätigkeit des Abrechnungsgerichtshofes, BGBl. 741. Die Auflösung des Gerichtshofs erfolgte im Oktober 1944 mit RFM Zl. V 5229/44. 
richtshof in $\S 56$ Abs. 1 VKSchG als "ordentliches Gericht" bezeichnet werde und seine Mitglieder nach Abs. 5 ,in der Ausübung ihres richterlichen Amtes" unabhängig seien, sodass er nicht als Verwaltungsbehörde angesehen werden könne. Die Frage der Natur des Abrechnungsgerichtshofs und unmittelbar im Zusammenhang damit jene nach der Zuständigkeit des VfGH bestimmte folglich die Beratungen. Gegen Neumann-Ettenreichs Auffassung wandte Hans Kelsen ein, dass es sich beim Abrechnungsgerichtshof in Wahrheit um ein Ausnahmsgericht handle. Unter einem Ausnahmsgericht im Sinne des (durch BGBl. 73/1968 aufgehobenen) Art. 83 Abs. 3 B-VG verstand man ein Gericht, welches nicht durch die geltenden Gerichtsorganisationsgesetze eingerichtet war; die Errichtung eines solchen war nur in den durch die Gesetze über das Verfahren in Strafsachen geregelten Fällen zulässig. Da es sich beim Abrechnungsgerichtshof nicht um ein Strafgericht handelte, hätte er nicht durch einfaches Bundesgesetz eingerichtet werden dürfen. Zur Debatte stand damit auch die Auslegung des Art. 83 Abs.3, nämlich dahingehend, ob Ausnahmsgerichte auch außerhalb des Strafrechts zulässig seien, und wenn ja, ob sie in diesem Fall durch einfaches oder durch Verfassungsgesetz zu errichten wären.

Gegen die Zurückweisung in nichtöffentlicher Sitzung angesichts dieser Unklarheiten sprachen sich außer Kelsen auch einige andere Mitglieder aus; ein entsprechender Antrag Stefan Falsers wurde jedoch mit knapper Mehrheit abgelehnt. Hinsichtlich des umstrittenen Art. 83 Abs. 3 erreichte Kelsen, dass eine Erklärung betreffend die Nichtanwendbarkeit desselben in die Begründung aufgenommen wurde: Durchgesetzt hatte sich letztlich eine einschränkende Auslegung, wonach sich diese Bestimmung nur auf Ausnahmsgerichte in Strafsachen bezog und das B-VG für die Errichtung sonstiger Ausnahmsge- richte keine besonderen Voraussetzungen vorsah. ${ }^{38}$

Somit wurde die Beschwerde zurückgewiesen und begründend ausgeführt: „1. Nach Artikel 144 B.V.G. hat der Verfassungsgerichtshof über Beschwerden wegen Verletzung verfassungsmäßig gewährleisteter Rechte nur zu erkennen, wenn die Verletzung durch die Entscheidung oder Verfügung einer Verwaltungsbehörde verursacht wurde. 2. Der Abrechnungsgerichtshof gehört nicht zu den Verwaltungsbehörden, er ist im §56 des Gesetzes vom 12. Juli 1921, B.G.B1. Nr. 393, als ordentliches Gericht bezeichnet. 3. Unter den Ausnahmegerichten, von denen Absatz 3 des Artikels 83 B.V.G. spricht, sind nur solche in Strafsachen $\mathrm{zu}$ verstehen; diese Gesetzesstelle hat keine Anwendung auf Gerichte in Zivilrechtsangelegenheiten." 39 Der Umstand, dass ein Instanzenzug von einer Verwaltungsbehörde zu einem Gericht führte, wurde offensichtlich als völlig unproblematisch angesehen und war zu keinem Zeitpunkt Gegenstand der Diskussion.

\section{Ergebnis}

Aus diesen Ausführungen ergibt sich, dass aus der Sicht der ersten Generation von Verfassungsrichtern, die nach dem B-VG 1920 zu urteilen hatten, dieses den einfachen Gesetzgeber ermächtigte, Instanzenzüge von Verwaltungsbehörden zu ordentlichen Gerichten zu führen. Über die weitere Entwicklung gibt der schon mehrfach zitierte Aufsatz Ewald Wiederins detailliert und überzeugend Aufschluss, sodass hier kurze Hinweise ausreichen: Mit der Verfassungsnovelle 1929 erfolgte die Streichung von Art. 94 Abs. 2, begründet wahlweise mit dessen Redundanz oder Unklarheit. Außerdem wurde die Zuständigkeit der ordentlichen Gerichte als

\footnotetext{
${ }^{38}$ Beratungsprotokoll zu VfGH B 87/23.

${ }^{39}$ VfGH B 87/23 VfSlg A38/1924.
} 
Unzuständigkeitsgrund des VwGH aus Art. 131 entfernt und damit ein Bescheidprüfungsmonopol desselben geschaffen, abgeleitet wohlweislich nicht aus dem Trennungsgrundsatz nach Art. 94, sondern aus der ausschließlichen $\mathrm{Zu}$ weisung der Kompetenz an die Gerichtsbarkeit des öffentlichen Rechts. Erst ab 1954 begann der VfGH, aus Art. 94 ein Verbot gewaltenübergreifender Instanzenzüge abzuleiten, begründet mit Bedenken gegen die behauptete organisatorische Zusammenfassung von Verwaltungs- und Gerichtsbehörden als Ergebnis einer instanzenmäßigen Verflechtung. ${ }^{40}$ Hans Kelsens Feststellung von 1922, wonach die Erfüllung des von Art. 94 Abs. 1 B-VG Geforderten „verschiedener Grade fähig" sei und diese Bestimmung der Interpretation viel Raum biete, hatte sich damit als zutreffend erwiesen.

\section{Korrespondenz:}

Mag. Mag. Mag. Ramon Pils, DipTrans

Universität Wien

Institut für Rechts- und Verfassungsgeschichte Abt. KRGÖ

Strohgasse 45/2d, 1030 Wien, Österreich ramon.pils@univie.ac.at

\section{Abkürzungen:}

\begin{tabular}{|c|c|}
\hline 1. Rep & Erste Republik \\
\hline $\mathrm{BMF}$ & Bundesministerium für Finanzen \\
\hline GGrG & $\begin{array}{l}\text { Grundgesetz über die richterliche } \\
\text { Gewalt, StGBl. 38/1918 }\end{array}$ \\
\hline HG & Höchstgerichte \\
\hline MRang & Ministerratsangelegenheiten \\
\hline MR & Ministerrat Erste Republik \\
\hline MRP & Ministerratsprotokoll \\
\hline $\mathrm{OBh}$ & Oberste Behörden \\
\hline RFM & Reichsminister der Finanzen \\
\hline StGGrG & $\begin{array}{l}\text { Staatsgrundgesetz über die } \\
\text { richterliche Gewalt, RGBl. 144/1867 }\end{array}$ \\
\hline VKSchG & $\begin{array}{l}\text { Vorkriegsschuldengesetz, } \\
\text { BGBl. 393/1921 }\end{array}$ \\
\hline VwGHG & $\begin{array}{l}\text { Verwaltungsgerichtshofgesetz, } \\
\text { RGB1. 36/1876 }\end{array}$ \\
\hline
\end{tabular}




\section{Quellen:}

Beratungsprotokoll zu VfGH B 87/23, 26. 11. 1923/ 1. 12. 1923, ÖStA, AdR, OBh, HG, 1Rep, VfGH, Kart. 47.

BMF Zl. 111866/21, 17. 12. 1921, ÖStA, AdR, BMF, 1 Rep, Dept 17/Frieden, Kart. 84.

Departementsentwürfe vom 23.11.1920, 26.2. und 28. 5. 1921, Beilage zu BMF Zl. 47193/21, ÖStA, AdR, BMF, 1Rep, Dept 17/Frieden, Kart. 70.

Gutachten Kelsen (unveröffentlicht), 27. 2. 1922, Beilage zu BMF Zl. 111866/21, ÖStA, AdR, BMF, 1 Rep, Dept 17/Frieden, Kart. 84.

MRP 220, 28. 8. 1922, ÖStA, AdR, MRang, MR, 1. Rep.

RFM Zl. V 5229/44, 21. 10. 1944, ÖStA, AdR, BMF, 1 Rep, Dept 17/Frieden, Kart. 84.

\section{Literatur:}

Edmund BERNATZIK, Rechtsprechung und materielle Rechtskraft (Wien 1886).

Wilhelm BRAUNEDER, Österreichische Verfassungsgeschichte (Wien ${ }^{112009)}$.

Emmerich CORETH, Die Anfechtung von Verwaltungsbescheiden im ordentlichen Rechtsweg, in: Österreichisches Verwaltungsblatt 1 (1930) Nr. 18, 1-7; Nr. 19, 1-5.

Hans Kelsen, Georg Froehlich, Adolf MerkL, Die Bundesverfassung vom 1. Oktober 1920 (Wien 1922, ND Wien 2003).

N. N. [Ferdinand SAMITSCH], Wann ist die Correctur der verwaltungsbehördlichen Entscheidung oder Verfügung vor dem Civilrichter im Rechtswege, und wann mittels Beschwerdeführung vor dem Verwaltungsgerichtshofe anzustreben? Beitrag zu einem Interpretationsversuche betreffend Art. 15 des Staatsgrundgesetzes über die richterliche Gewalt von 21. December 1867, R-G-B1. Nr. 144, in: Oesterreichische Zeitschrift für Gesetzgebung und Rechtsprechung auf dem Gebiete der Verwaltungs-Rechtspflege 1 (1877) 1-21.

Robert Walter, Die Entstehung des BundesVerfassungsgesetzes 1920 in der Konstituierenden Nationalversammlung (Wien 1984).

Ewald WIEDERIN, In allen Instanzen getrennt - Zum Verhältnis von Justiz und Verwaltung am Beispiel des strafprozessualen Vorverfahrens, in: Vienna Law Inauguration Lectures II (Wien 2010) 41-74. 